\title{
Temporal changes in the gonococcal serovar patterns in Stockholm during two years with special reference to PPNG strains
}

\author{
A-K Rudén
}

\begin{abstract}
Objective-To analyse temporal changes in gonococcal serovar patterns in Stockholm during a two year study period (1987-1989) to elucidate the dynamics of gonorrhoea epidemiology. Design-The study population comprised 857 patients with culture proven gonorrhoea and with serotyped gonococcal isolates. The probable geographical origin of the infection was determined in 690 of the patients.

Results-A total of $108 \mathrm{Ph} / \mathrm{GS}$-serovars were identified. Most $(73 \%)$ of the serovars were recognised only during one or two quarters of the study period and comprised $16 \%$ of the isolates. Seven serovars were encountered during all eight quarters. Three of these serovars i.e. Arost/Aedgkih (IA-1, IA-2), Bropt/Bajk (IB-3, IB-6), Brpyust/Bacejk (IB-1, IB-2) were the most prevalent in the overall study, accounting for $60 \%$ of the isolates during the first quarter of the study and $36 \%$ of the isolates during the last quarter. Fifty-seven percent of the patients were infected in Stockholm (endemic infection). The proportion of endemic isolates among the three most common serovars declined throughout the study period (77\% during the first quarter; $47 \%$ during the last quarter). A total of $32 \mathrm{Ph} / \mathrm{GS}$-serovars were recognised among 80 PPNG strains. Only four of these 32 serovars were encountered during more than two quarters. Of 57 patients with PPNG strains and with geographical origin of the infection known, only seven (12\%), all infected in Sweden, might have transmitted their infection further into the society.
\end{abstract}

Conclusions-The decline in the total number of gonorrhoea cases seen in Stockholm during the study period, was due mainly to a decline of endemic isolates of the three most prevalent serovars. Results from contact tracing and serotyping indicated that PPNG infections acquired abroad seldom become established in the community. Serovar determination seems valuable mainly as a tool for surveillance whereby the introduction and circulation of gonococcal strains in the community can be pursued.

(Genitourin Med 1994;70:256-261)
Introduction

The development of a reliable and well functioning classification system for Neisseria gonorrhoeae by using monoclonal antibodies against protein $\mathrm{I}$, the major outer membrane protein, has provided us with a tool for interpreting gonorrhoea epidemiology at the community level. ${ }^{1-6}$

In 1987, we started a two year project with the aim of "eradicating" endemic gonorrhoea in Stockholm. At that time the incidence of gonorrhoea in Sweden had been continuously decreasing since 1970, except for a minor peak in 1976. The Stockholm area accounted for about one third of all Swedish gonorrhoea cases, $5.4 \%$ of which were caused by betalactamase producing Neisseria gonorrhoeae (PPNG) strains.

One object of the project was to characterise endemic versus non-endemic gonorrhoea in order to identify groups at risk for transmission of the infection. This would also include an evaluation of the effects of intensified contact tracing performed by specially trained social workers. ${ }^{7}$ Another object was to correlate antibiotic susceptibility, serovars, and auxotypes of the gonococcal isolates to geographical origin of the infection. ${ }^{8}$

The aim of the present study was to describe and analyse temporal changes in serovar patterns in the community to show the dynamics in the spread of Neisseria gonorrhoeae strains with special reference to PPNG strains.

\section{Methods}

The aim of the whole project was to eradicate endemic gonorrhoea from Stockholm. Endemic infection is therefore defined as infection acquired in Stockholm and non-endemic infection as infection acquired abroad (imported) or from other parts of Sweden.

Study design The study group comprised all patients in Stockholm with culture proven gonorrhoea during the two year study period (October 1987-September 1989), diagnosed at any of the six bacteriological laboratories and with serologically classified gonococcal isolates. Strains from four patients were not serotyped and these patients were excluded. A patient returning within six weeks with a gonococcal isolate of identical serovar to that isolated on the first occasion and with no negative culture in between was only counted once. Our aim was to see all patients at one or other of the four venereal outpatient clinics in 
Stockholm. Doctors working outside the venereal clinics were asked to refer patients (with the patient's consent) to one of the venereal clinics for treatment, test-of-cure culture and contact-tracing interview performed by a social worker.

The two year study period was divided into quarters with October-December 1987 defined as quarter number 1, January-March 1988 as quarter number 2 etc. For a more detailed description of the principles of design of the study see reference number 7 .

Study poulation Out of 857 patients with gonorrhoea, included in the study, $671(78 \%)$ attended a venereal clinic for a follow-up visit with a contact-tracing interview. The remaining 186 patients did not attend any venereal clinic.

Contact tracing All 671 patients were subjected to a semi-structured interview, performed by social workers. The questions included the number and identity of sexual partners during the previous six months, the probable source of infection, the likely geographical origin of the infection and the existence of a steady partner with whom the patient had an ongoing sexual relationship.

Microbiological methods Specimens were obtained from the urethra in men and from the urethra and cervix in women. Rectal and pharyngeal specimens were obtained when appropriate. The strains were cultured and identified as described earlier. ${ }^{8}$

All strains were classified serologically with co-agglutination into serogroups (WI and WII/III) and serovars as previously described. ${ }^{19}$ Two different sets of monoclonal antibody reagents were used: the $\mathrm{Ph}$-panel ${ }^{510}$ (Pharmacia Diagnostics AB, Uppsala, Sweden) and the GS-panel ${ }^{1}$ (Syva, Palo Alto, USA). For the choice of monoclonal antibodies, nomenclature used for the designation of serovars and correlations between the $\mathrm{Ph}$ - and the GS-serovars see Bygdeman et al..$^{5}$ The corresponding serovar nomenclature according to Knapp et $a l^{2}$ is given in brackets. A serovar combination of a $\mathrm{Ph}$-serovar and a GS-serovar is in this paper referred to as a $\mathrm{Ph} / \mathrm{GS}$-serovar or just a serovar.
Before the start of the study laboratory technicians from all six bacteriological laboratories in Stockholm were educated and trained in the serological classification technique although three of the six laboratories already had used the technique routinely for several years. If the laboratory technician had any problem with interpretation of the co-agglutination results, one laboratory (Huddinge Hospital) had a supervising role. Every new or unusual serovar or serovar combination of the two panels was doublechecked at this laboratory.

All isolates were typable. If a patient had strains of the same serovar, isolated at the same time from several sites, only one strain was included in the study.

Statistical analysis Chi square test with Yates' correction was used.

\section{Results}

General Among the 857 patients (332 women and 525 men) comprising the study population a total of 859 gonococcal strains were isolated. Two patients had a double infection with two strains of different $\mathrm{Ph} / \mathrm{GS}$ serovars isolated from the urethra. A probable geographical origin of the infection was determined for $690(81 \%)$ of the patients. Of these 690 patients, $57 \%$ were considered to have an endemic infection and $43 \%$ to have a nonendemic infection.

Out of the 859 isolates, $231(27 \%)$ belonged to serogroup WI and $626(73 \%)$ to serogroup WII/III. Two isolates from two women reacted with both WI and WII/III reagents (WI-WII/III) and belonged to the same serovar.

Serovar determination A total of $108 \mathrm{Ph}$ GS-serovars was encountered. Table 1 shows the distribution of these serovars by quarter through the study period. The majority ( $86 \%)$ of the $\mathrm{Ph} / \mathrm{GS}$-serovars belonged to serogroup WII/III (table 2). Forty-three percent $(6 / 14)$ of the WI serovars and $61 \%(57 / 93)$ of those of serogroup WII/III were confined to only one gender $(p=0 \cdot 3)$. Forty-one percent of all serovars were recognised in isolates from both

Table 1 Distribution of 859 isolates into $108 \mathrm{Ph} / G S$-serovars during the 8-quarter study period

\begin{tabular}{|c|c|c|c|c|c|c|c|c|c|c|}
\hline \multirow[b]{3}{*}{ Serovars } & & \multicolumn{8}{|c|}{ Number of isolates by quarter } & \multirow{3}{*}{$\begin{array}{l}\text { Total } \\
\text { number }\end{array}$} \\
\hline & & \multicolumn{8}{|c|}{ Quarter number } & \\
\hline & & 1 & 2 & 3 & 4 & 5 & 6 & 7 & 8 & \\
\hline Arost/Aedgkih & (IA-1, IA-2) & 37 & 14 & 29 & 24 & 17 & 16 & 16 & 11 & 164 \\
\hline Bropt/Bajk & (IB-3, IB-6) & 16 & 30 & 32 & 22 & 10 & 15 & 14 & 8 & 147 \\
\hline Brpyust/Bacejk & (IB-1, IB-2) & 29 & 17 & 7 & 15 & 18 & 9 & 9 & 8 & 112 \\
\hline Arst/Aedih & (IA-6) & 6 & 2 & 3 & 4 & 2 & 6 & 8 & 2 & 33 \\
\hline Bpyvut/Beghjk & (IB-4, IB-11) & 3 & 5 & 7 & 1 & 6 & 4 & 4 & 3 & 33 \\
\hline Bropyst/Back & (IB-1, IB-2) & 2 & 3 & 1 & 9 & 3 & 6 & 3 & 1 & 28 \\
\hline Bropst/Bacjk & (IB-1, IB-2) & 1 & 3 & - & 1 & 8 & 6 & 3 & 1 & 23 \\
\hline Bropt/Bacjk & (IB-1, IB-2) & 5 & 2 & 4 & 3 & 5 & 2 & 1 & - & 22 \\
\hline Bopt/Bajk & (IB-3, IB-6) & 3 & 2 & 3 & - & - & 2 & 3 & 3 & 16 \\
\hline Bpyust/B́cegik & (IB-5, IB-7) & 2 & 2 & 1 & 1 & 2 & 1 & 2 & 2 & 13 \\
\hline Bropyt/Bak & (IB-3, IB-6) & - & - & 2 & 3 & $\overrightarrow{1}$ & 2 & 3 & 1 & 12 \\
\hline Bopyt/Bak & (IB-3, IB-6) & 4 & 1 & 1 & 2 & - & - & 1 & 2 & 11 \\
\hline Bopst/Bcgik & (IB-5, IB-7) & - & 1 & - & 2 & 1 & 3 & 2 & 1 & 10 \\
\hline $\begin{array}{l}\text { Brpyut/Bacejk } \\
\text { Additional } 94\end{array}$ & (IB-1, IB-2) & 2 & - & 1 & - & 3 & 4 & - & - & 10 \\
\hline $\mathrm{Ph} / \mathrm{GS}$-serovars* & & 26 & 25 & 19 & 25 & 27 & 41 & 30 & 32 & 225 \\
\hline Total & & 136 & 107 & 110 & 112 & 103 & 117 & 99 & 75 & 859 \\
\hline
\end{tabular}

* Serovars with less than 10 isolates each. 
Table 2 Distribution of $108 \mathrm{Ph} / \mathrm{GS}$-serovars among 859 gonococcal isolates in relation to serogroup and sex

\begin{tabular}{lcll}
\hline & \multicolumn{3}{l}{ Serogroup } \\
\cline { 2 - 4 } No of serovars in & $W I$ & WII/III & WI-WII/III \\
\hline female isolates only & 1 & 11 & 1 \\
male isolates only & 5 & 46 & - \\
isolates from both sexes & 8 & 36 & - \\
Total & 14 & 93 & 1 \\
\hline
\end{tabular}

Table 3 Transient Ph/GS-serovars versus those seen during the whole of the study period. Number of isolates in relation to geographical origin of the infection

\begin{tabular}{lcrcr}
\hline $\begin{array}{l}\text { No of quarters } \\
\text { serovars } \\
\text { were seen }\end{array}$ & $\begin{array}{l}\text { No of } \\
\text { servars }\end{array}$ & (\%) & $\begin{array}{l}\text { No of } \\
\text { isolates }\end{array}$ & (\%) \\
\hline 1 & 61 & $(56)$ & 77 & $(9)$ \\
2 & 18 & $(17)$ & 60 & $(7)$ \\
3 & 4 & $(4)$ & 16 & $(2)$ \\
4 & 8 & $(7)$ & 50 & $(6)$ \\
5 & 5 & $(2)$ & 16 & $(2)$ \\
6 & 3 & $(5)$ & 57 & $(7)$ \\
7 & 7 & $(3)$ & 53 & $(6)$ \\
8 & 108 & $(100)$ & 530 & $(62)$ \\
Total & & & &
\end{tabular}

sexes, $47 \%$ in male isolates only and $12 \%$ exclusively in female isolates (table 2).

Most (73\%) of the serovars were encountered only during one or two quarters of the study. They accounted for $16 \%$ of the isolates. Thus, a minority, $27 \%$ of the serovars, represented $84 \%$ of the isolates and were seen during 3 quarters or more (table 3 ).

Seven serovars $(6 \%)$ with 530 isolates, that is $62 \%$ of all isolates were recognised during all 8 quarters (table 3 ). Three of these serovars (Arost/Aedgkih (IA-1, IA-2), Bropt/Bajk (IB-3, IB-6) and Brpyust/Bacejk (IB-1, IB-2)) accounted for $49 \%(423 / 859)$ of all isolates in the study. During the first quarter of the study period, $60 \%(82 / 136)$ and during the last quarter, $36 \%(27 / 75)$ of all isolates belonged to these serovars $(p<0.005)$. The decline in total number of isolates from the first quarter to the last (table 4) was due mainly to a decline in number of isolates of these three serovars (fig 1). The proportion of endemic isolates among these serovars was $65 \%$ $(229 / 355)$ of those with known geographical origin. During the first quarter of the study period, $77 \%(54 / 70)$ of the isolates were of endemic origin with a decrease to $47 \%(9 / 19)$ during the last quarter (fig 2). Thus, the decrease in number of isolates of these three serovars was due mainly to a lower number of endemic isolates.

The remaining four serovars (Arst/Aedih (IA-6), Bpyvut/Beghjk (IB-4, IB-11), Bropyst/

Table 4 Number of Ph/GS-serovars, number of isolates and the ratio between these variables by quarter

\begin{tabular}{llll}
\hline $\begin{array}{l}\text { Quarter } \\
\text { no }\end{array}$ & $\begin{array}{l}\text { No of } \\
\text { serovars }\end{array}$ & $\begin{array}{l}\text { No of } \\
\text { isolates }\end{array}$ & Ratio \\
\hline 1 & 29 & 136 & $0 \cdot 21$ \\
2 & 32 & 107 & $0 \cdot 30$ \\
3 & 27 & 110 & $0 \cdot 25$ \\
4 & 27 & 112 & $0 \cdot 24$ \\
5 & 36 & 103 & $0 \cdot 35$ \\
6 & 41 & 117 & $0 \cdot 35$ \\
7 & 34 & 99 & $0 \cdot 34$ \\
8 & 33 & 75 & $0 \cdot 44$ \\
\hline
\end{tabular}

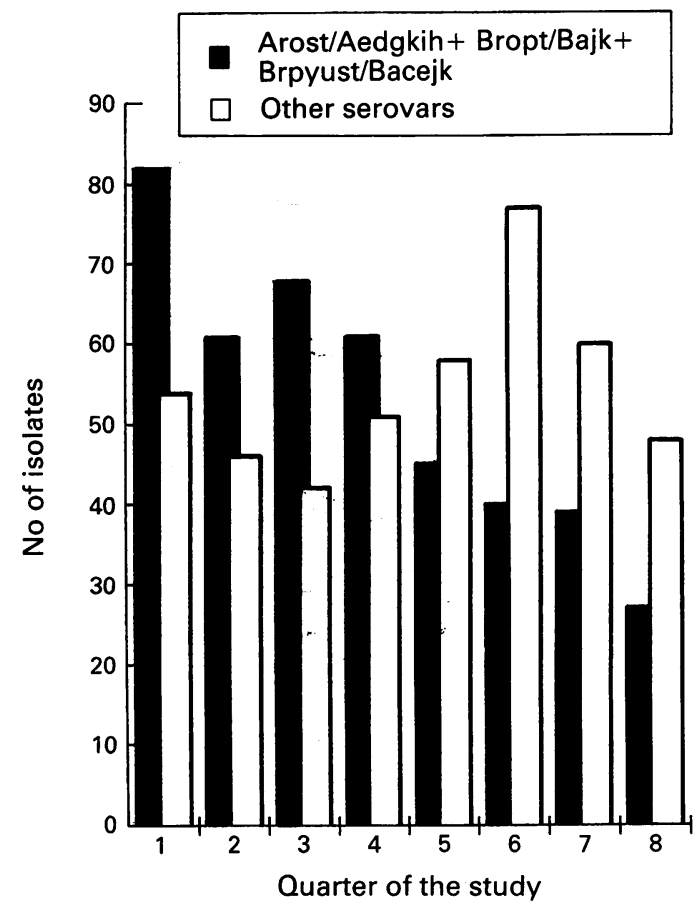

Figure 1 Number of isolates by quarter in relation to isolates of the three dominating Ph/GS-serovars; Arost/Aedgkih (IA-1, IA-2), Bropt/Bajk (IB-3, IB-6), Brpyust/Bacejk (IB-1, IB-2) and isolates of other serovars.

Back (IB-1, IB-2), Bpyust/Bcegik (IB-5, IB7)) of those identified during all eight quarters, accounted for $13 \%(108 / 859)$ of all isolates; $10 \%(13 / 136)$ of the strains isolated during the first quarter and $12 \%(9 / 75)$ during the last quarter. Each of these serovars was represented mainly by non-endemic isolates. This inflow of non-endemic strains showed a relatively stable pattern during the study period. Among the remaining 101 serovars none became dominant over the study period.

An increasing diversity was identified during the study period between number of serovars and number of isolates, caused by a minor increase in the number of serovars recognised per quarter during the study and a major decrease in the number of isolates (table 4).

Of all patients in the study group, $80(9 \%)$ (14 women and 66 men) had an infection with a PPNG strain. Among the 80 PPNG strains $32 \mathrm{Ph} / \mathrm{GS}$-serovars were seen; $4 \mathrm{WI}$, $27 \mathrm{WII} / \mathrm{III}$ and 1 WI-WII/III. Eighty-four percent of the PPNG strains belonged to serogroup WII/III. There was no dominating serovar but Arst/Aedih (IA-6) was the most commonly encountered with $13(16 \%)$ isolates from 13 patients. PPNG strains of this serovar was identified during five quarters of the study period. In the overall study, Arst/Aedih (IA-6) accounted for 33 strains. Thus, $39 \%$ of all were PPNG strains. PPNG strains of the second most common serovar Bpyvut/Beghjk (IB-4, IB-11) with 11 isolates (14\%) was represented during six quarters. Most of the serovars, $28(87.5 \%)$ out of 32 , were found only during one or two quarters.

The ratio between the number of serovars 


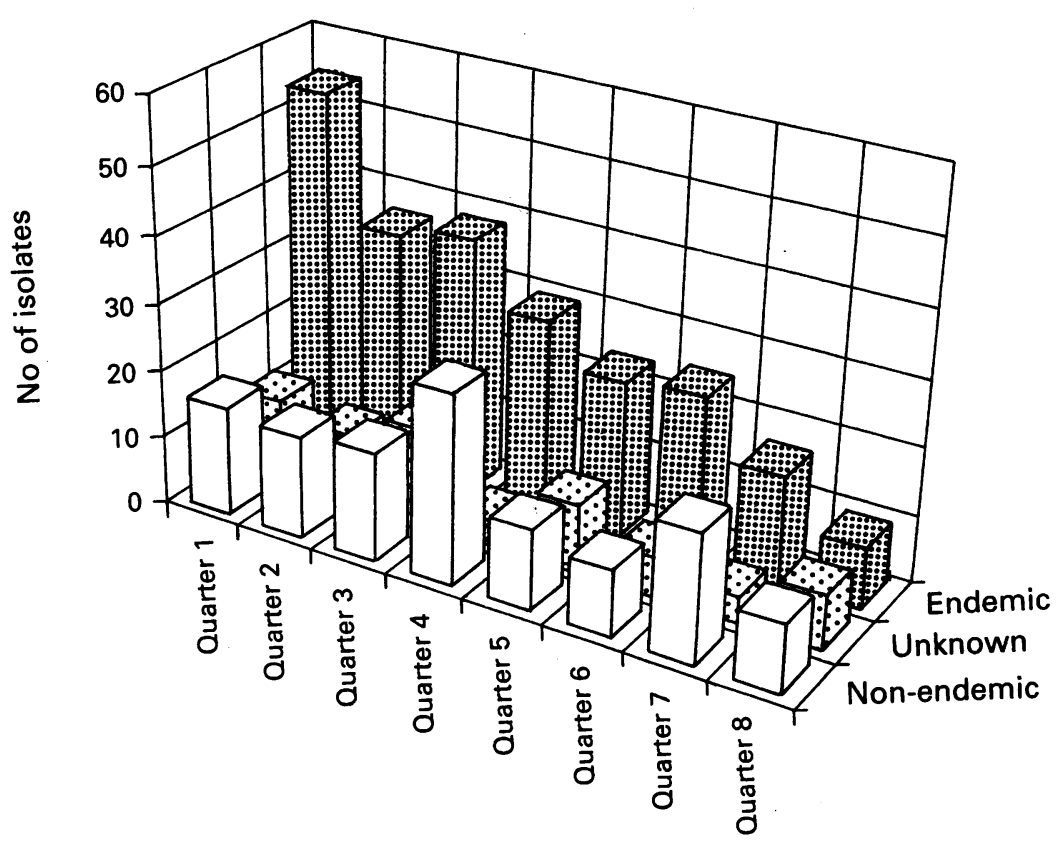

Figure 2 Distribution by quarter of isolates belonging to the three dominating Ph/GSserovars in relation to endemic infection, non-endemic infection and infection of unknown origin. ing had sex with two unknown female partners. During the study period only four PPNG strains of this serovar were recognized, all isolated from men.

One woman infected in Stockholm admitted to sexual contacts with three partners. She reported that she had informed two of them of the suspicion of gonorrhoea but refused to identify them. The remaining partner was examined and was found to be infected. This man denied additional sexual partners. The PPNG strains involved belonged to the second most common serovar among these strains, Bpyvut/Beghjk (IB-4, IB-11). Of the additional nine patients, all men, with PPNG strains of this serovar, four had acquired their infection abroad and denied sexual contact after returning to Sweden. The remaining five patients were not subjected to contact tracing at a venereal clinic.

\section{Discussion}

The aim of the present study was to describe and analyse changes over time in serovar patterns in the community. Two different sets of monoclonal antibodies (Ph- and GS-panels) were used to improve the resolution. Earlier studies have shown that the resolution increases when combining these two sets of monoclonal reagents. ${ }^{511}$

In two previous studies in Stockholm in $1979-80^{12}$ and in $1982-83,{ }^{13} 42 \%$ and $33 \%$ of the strains, respectively, belonged to serogroup WI compared with $27 \%$ in the present study. Thus, a progress towards a greater dominance of WII/III strains was noted. This is unfortunate since WII/III strains more often show decreased susceptibility to different antibiotics compared with WI strains. ${ }^{514}$ Bygdeman et $a l^{8}$ have shown that the high sensitivity to antibiotics among WI strains is correlated with one WI serovar, Aedgkih, which in several studies have accounted for the majority of WI strains. ${ }^{5}$ In the present study, strains of this serovar markedly decreased in number during the study period.

A greater heterogeneity of serovars was seen in serogroup WII/III compared with serogroup WI (table 2). This is in accordance with earlier studies. ${ }^{511}$

Almost half (47\%) of the 108 serovars observed were encountered in isolates from men only (table 2 ). The corresponding figure for women was $12 \%$. In the study in Stockholm 1982-83 mentioned above, ${ }^{13}$ an almost identical picture was noted with $47 \%$ of the serovars found in isolates from men only and $11 \%$ in isolates from women. We have shown earlier that men more often acquired their infection outside Stockholm compared with women ${ }^{7}$ and furthermore that imported strains frequently belong to serovars that are unusual in Stockholm. ${ }^{13}$

Sixteen percent of the strains showed a transient pattern that was encountered only during one or two quarters of the study period (table 3). These isolates represented the major part $(73 \%)$ of the 108 serovars.

Only seven serovars were persistent Stockholm. Out of the four patients infected in Stockholm, three had unique serovars, not seen in any other PPNG isolates. The fourth patient, a man, had a strain with the serovar Arost/Aedgkih (IA-1, IA-2) and reported hav- 
throughout all eight quarters (table 3 ). Three of these serovars accounted for almost half of the isolates. This is in accordance with previous studies from other geographical areas using the $\mathrm{Ph} / \mathrm{GS}$-panels. ${ }^{515}$ A majority (65\%) of the isolates of these three serovars was acquired in Stockholm and constituted the basis for endemic infections. From the first to the last quarter of the study, the proportion of isolates belonging to these three most prevalent serovars, markedly declined from $60 \%$ to $36 \%$ without a corresponding decline in the proportion of isolates of other serovars (fig 1). In a five year study in Edinburgh, Scotland, a similar decline was seen in the total number of gonorrhoea cases. ${ }^{16}$ Fifty-eight percent of the infections were acquired in Edinburgh, 33\% in the rest of the United Kingdom and 5\% abroad. The same three GS-serovars dominated as in our study. Isolates of two of these serovars, Aedgkih (IA-1, IA-2), Bacejk (IB-3, IB-6) decreased in frequency in parallel with the overall fall in the prevalence of gonorrhoea. However, one serovar, Bacejk (IB-1, IB-2), was persistent at a constant level. No information was given about the proportion of endemic infections in these serovars. The decrease in the number of gonococcal isolates identified throughout the study period in the present study was mainly due to a decrease among endemic isolates of the three dominating serovars (fig 2).

In a previous study, enrolling 671 of the 859 patients in the present study, we showed by using contact tracing that strains acquired outside Stockholm mostly did not establish themselves in the community. ${ }^{7}$ Furthermore, a decrease from $75 \%$ to $40 \%$ in the proportion of endemic infection was noted during the two year study period. Usually, patients with non-endemic infection did not transmit their infection after their return to Stockholm. Alternatively the infection ended as a second generation imported infection with no further transmission. Thus, we have a considerable inflow of strains that do not become established as endemic strains. ${ }^{7}$

The three most prevalent serovars with a dominance of endemic isolates were persistent throughout the study period. The major part of the serovars, however, showed a transient pattern. The reappearance of a serovar could depend on reintroduction either from an external source or from an earlier undetected case, probably with asymptomatic infection. Furthermore, serovar identity of two strains does not ensure that they have the same clonal origin, especially if they belong to the most prevalent serovars or are of different geographical origin.

The proportion of PPNG strains in the study was $9 \%$, with a significant difference between the genders. Out of the $108 \mathrm{Ph} / \mathrm{GS}$ serovars seen in the whole study of 859 isolates, $32(30 \%)$ were identified among the 80 PPNG isolates. Only four of these 32 serovars were recognised during more than two quarters suggesting that the major part of the strains were imported. This conclusion was supported by contact tracing data revealing that $75 \%$ of the 57 patients with probable geographical origin of the infection determined, had acquired their infection abroad.

The most common serovar combination among PPNG strains in the present study was Arst/Aedih (IA-6) representing $16 \%$ of all PPNG strains. In a study in 1982-1983 in Sweden comprising 253 PPNG strains, Arst/Aedih (IA-6) was one of the most prevalent serovars accounting for $20 \%$ of the isolates. ${ }^{17}$ In a study from Amsterdam during two time periods, 1981-1982 and 1985, Arst/Aedih (IA-6) was the only common serovar among PPNG strains on both occasions. ${ }^{6}$

In the present study we studied the dynamics and transmission of PPNG strains in the society by analysing results of serovar determination and contact tracing in conjunction. The 43 patients with PPNG strains, acquired abroad and who were contact traced at one of the venereal clinics, either did not transmit their infection into the society at all or did only transmit the infection to their steady partner where the transmission ended. Seven of the 14 patients infected with PPNG strains in Sweden, had unknown partners only. Why did we not find possible partners with isolates of the same serovars to these patients infected in Stockholm and with unknown partners? Alternative explanations are possible. The partner was examined outside Stockholm, or outside the study period. The partner had an asymptomatic infection or had typical symptoms and was treated for gonorrhoea without a preceding culture taken. He or she had practised self-medication or had spontaneously healed. Even though every male patient was asked about sexual orientation both by a social worker and a physician, independently, there might have been an incorrect answer. Furthermore, the laboratory might have produced a false negative result. Although the sensitivity of gonococcal cultures is high, false negative results exist. Another possibility would be that the PPNG strain had lost its plasmid encoding for beta-lactamase production. A false interpretation of the co-agglutination results might also have been made in the laboratory. However, every new or unusual serovar was double-checked.

With serovar analysis and contact tracing data we have shown that the number of strains belonging to endemic serovars has continuously declined and endemic infections are becoming uncommon in Stockholm.

1 Tam MR, Buchanan TM, Sandström EG, et al. Serological classification of Neisseria gonorrhoeae with monoclonal antibodies. Infect Immun 1982;36:1042-53. 2 Knapp JS, Tam MR, Nowinski RC, Holmes KK, Sandström EG. Serological classification of Neisseria gonorrhoeae with use of monoclonal antibodies to gonogonorrhoeae with use of monoclonal antibodies to gono-
coccal outer membrane protein I. $¥$ Infect Dis 1984;150: 44-8.

3 Ramstedt KM, Hallhagen GJ, Bygdeman SM, et al. Serologic classification and contact-tracing in the control of microepidemics of $\beta$-lactamase-producing Neisseria gonorrhoeae. Sex Transm Dis 1985;12:209-14

4 Knapp JS, Holmes KK, Bonin P, Hook III, EW. Epidemiology of gonorrhea: distribution and temporal 
changes in auxotype/serovar classes of Neisseria gonorrhoeae. Sex Transm Dis 1987;14:26-32.

5 Bygdeman S. Polyclonal and monoclonal antibodies applied to the epidemiology of gonococcal infection. In: Young H, McMillan A, eds. Immunological Diagnosis of Sexually Transmitted Diseases. New York: Marcel Dekker Inc, 1988:117-65.

6 Ansink-Schipper MC, Bygdeman SM, van Klingeren B, Sandström EG. Serovars, auxotypes, and plasmid profiles of PPNG strains with Asian type plasmid isolated in Amsterdam. Genitourin Med 1988;64:152-5.

7 Rudén A-K, Jonsson A, Lidbrink P, Allebeck P, Bygdeman S. Endemic versus non-endemic gonorrhoea in Stockholm: results of contact tracing. Int $\mathcal{F}$ STD AIDS 1993;4:287-92.

8 Bygdeman SM, Rudén A-K, Jonsson A, et al. Antibiotic susceptibility, serovars and auxotypes of gonococcal isolates in Stockholm. Relation to geographical origin of the infection. Int ₹ STD AIDS 1993;4:33-40.

9 Sandström E, Danielsson D. Serology of Neisseria gonorrhoeae. Classification by co-agglutination. Acta Pathol Microbiol Scand Sect B 1980;88:27-38.

10 Sandström E, Lindell P, Härfast B, Blomberg F, Rydén A-C, Bygdeman S. Evaluation of a new set of Neisseria gonorrhoeae serogroup W-specific monoclonal antibodies for serovar determination. In: Schoolnik GK, Brooks GF, Falkow S, et al. The Pathogenic Neisseriae. Washington, DC: American Society for Microbiology, 1985, 26-30.
11 Sandström E, Tam M, Bygdeman S. Antigenic drift of gonococcal protein I as judged by serovar determination. In: Schoolnik GK, Brooks GF, Falkow S, et al. The Pathogenic Neisseriae. Washington, DC: American Society for Microbiology, 1985, 13-9.

12 Bygdeman S, Danielsson D, Sandström E. Serologica classification of Neisseria gonorrhoeae by co-agglutination: A study of serological patterns in two geographical aton: A Studeden. Acta Derm Venereol (Stockh) 1981;61:423-7. of Sweden. Acta Derm Venereol (Stockh) 1981;61:423-7. Rudén A-K, Bäckman M, Bygdeman S, Jonsson A, Ringertz O, Sandström E. Analysis of serovar distribution as a tool in epidemiological studies in gonorrhoea. Acta Derm Venereol (Stockh) 1986;66:325-33.

14 Bygdeman S. Antibiotic susceptibility of Neisseria gonorrhoeae in relation to serogroups. Acta Pathol Microbio Scand Sect B 1981;89:227-37.

15 Young H, Moyes A, Robertson DHH, et al. Gonococcal infection within Scotland: Antigenic heterogeneity and antibiotic susceptibility of infecting strains. Eur $f$ Epidemiol 1990;6:1-8.

16 Young H, Moyes A, Ross J, McMillan A. A serovar analysis of heterosexual gonorrhoea in Edinburgh 1986-90. Genitourin Med 1992;68:16-9.

17 Bygdeman SM, Gillenius E-C, Sandström EG. Comparison of two different sets of monoclonal antibodies for the serological classification of Neisseria gonorrhoeae. In: Schoolnik GK, Brooks GF, Falkow S, et al. The Pathogenic Neisseriae. Washington, DC: American The Pathogenic Neisseriae. Washington,
Society for Microbiology, 1985:31-6. 